\title{
An Object-Oriented Environment for the Construction Planning of High-Rise Buildings
}

\author{
Masaaki Ikeda*, Amr A. Oloufa", and Yasunari Sekihara"
}

* Fujita Corporation, CIC Project Team, Sendagaya 5-23-15, Fujita Daisan-biru, Shibuya-ku, Tokyo 151, Japan.

** The Pennsylvania State University, Department of Architectural Engineering, 104 Engineering Building "A", University Park, PA 16802, USA.

\begin{abstract}
New buildings are experiencing a continuous increase in the sophistication of their building systems and methods of construction. This situation creates a need for a planning tool that is capable of serving one or more of the complex set of activities during the project life phases.

Several researchers have devised methods for the modeling of buildings in an attempt to develop a representation that is capable of supporting a collaborative environment for engineering and construction. Some of these modeling approaches attempt to enable the user to use the same model for conceptual and detailed design, while others target primarily the construction activities. Other models have also attempted to cover the design and construction interface.

This paper will describe a modeling environment for the construction planning of high rise buildings. Issues covered include the development of an object-oriented CAD environment that enables the construction planner to evaluate potential construction sequences using both graphical, attribute, and knowledge-based inputs. The manuscript will conclude with other approaches described in the literature, problems that have limited their implementation, then extensions to this research.
\end{abstract}

\section{INTRODUCTION}

Advanced applications in architectural engineering involve the use of computer graphics. For example, In Construction Engineering, computer graphics are used to study project scenarios for facilities planning. In HVAC Engineering, graphics are used to evaluate building designs in terms of their energy efficiency, fire safety considerations and ventilation modeling. In Illumination Engineering, graphics are used to visualize the engineering and aesthetic aspects of light components and their impact on the quality of 
life in buildings. In Structural Engineering, graphics are used in automatic mesh generation, and visualization of deformations.

There is an ever-increasing need for better integration between design and construction. This effort can be vastly improved through the utilization of "intelligent drawings" which are created by interfacing the graphics model with an external database that stores information related to the project components. By polling this database, designers and builders have access to all the information needed for the development of the project through its various phases.

Developing "smarter" models allows the designers and contractors to utilize the same computer model for design, estimating, planning and control of projects. This increases the quality of the project through better communications, and decreases the time needed for design coordination and project changes.

\section{WHAT IS CIC?}

Computer Integrated Construction (CIC), is a concept that is similar to CIM (Computer Integrated Manufacturing), which is widely employed in the manufacturing industry. CIC concepts and products are intended to be used in the various stages of construction such as planning, design, estimating, construction, and operations, to improve work efficiency and productivity. ClC enables all divisions of a construction company to have access to project-related information. It also allows work which is performed in a sequential manner to be executed simultaneously and cooperatively thereby reducing project duration and improving efficiency and productivity.

In Japan, economic stagnation in the last few years in addition to government deregulation has intensified cost competition within the Japanese construction industry. Until recently, research and development conducted by the government and general contractors concentrated on the development of construction machinery and new building materials, reflecting the emphasis on the development of new construction methods. This research emphasis grew during the recent economic boom when skilled workers were in high demand. However, while productivity of the construction site operations improved as a result of these research efforts, productivity in other areas such as planning, design, and estimating have not kept pace. With the current emphasis on re-engineering and the improvement of productivity of white-collar jobs, CIC has been recognized in the Japanese construction industry as a highly effective tool in increasing productivity.

A main ingredient for the success of CIC activities is the development of a powerful and flexible data model. However, this is a cumbersome task since (i) abstractions of buildings vary significantly depending on the their purpose such as architectural design, structural design, estimating, or construction; and (ii) the information is constantly changing and evolving as the project progresses; and (iii) there is a need for better standards for information exchange between contractors.

\section{CIC RESEARCH IN FUJITA}

Fujita Corporation, a leading general contractor in Japan, sought to apply the products of CIC research to middle and high-rise steel-frame reinforced concrete buildings constructed using the HPC method. The HPC method is a system that utilizes precast concrete components for shear walls, nonbearing walls, slabs, balconies, corridors and other building elements. This method was selected because it has a 30 -year history of successful application in the construction industry. 
The CIC team carried out several important assignments for HPC construction projects, including (1) examination of existing work systems, (2) collection of performance information on construction projects conducted in the past, (3) studies on methods to make use of planning charts developed with 2D CAD for construction management, (4) examination of methods to employ EDI (Electric Data Interchange) for exchanging building information with architectural design, and component manufacturing companies, and (5) using three-dimensional $\mathrm{CAD}$, development of software that can share files containing building information.

In August 1991, Fujita and the Pennsylvania State University signed an agreement for joint research. In the first year of the project, the team analyzed internal work procedures and information flow between work divisions. In the second year of the project, the team focused its efforts on the development of building product models, experimenting with a building modeling system to be used in an object-oriented environment, and a preliminary construction planning system using this building model system. In the third and fourth years, the research developed a soil information system for use in shield tunnel construction, as well as an object-oriented simulation system for shield tunneling projects. Fujita has also concurrently carried out a study on object-oriented development as discussed later in this paper, and intends to address the challenge of developing an integrated project database applicable to future building construction.

CIC activities focused on the development of a framework comprised of a family of products that share information. This output from the structural design process is manipulated further by other products that support construction planning and monitoring. Using this framework, CIC's primary objective of joint ownership of information can be achieved. The following paragraphs outline the major products that are developed under this framework (see Figure 1).

\section{(1) Structural Planning System (FIND)}

This system is designed to estimate sectional properties of construction components and to develop detailed structural designs using other conventional structural analysis systems.

\section{(2) Building Product Model Input System (FINE-MODELER)}

This is a system by which building product data can be output in a standard format, and a complete three-dimensional building product model can be developed by specifying building components.

\section{(3) Preliminary Construction Planning System (FINE-PLANNER)}

This system checks if there are problems in preliminary design following an examination of the planned construction method. Work data and/or resource data produced at this phase may be input into the construction management system and/or the estimating system.

\section{(4) Construction Management System (PF-NETS)}

This is a scheduling system that can be used to develop a schedule based on the data produced by FINE-PLANNER, thus supporting detailed construction planning and progress monitoring.

\section{(5) On-Site Information Collection System}

This system, which can be run on palmtop computer at the construction site. It supports the recording of instructions for daily activities with the aid of the project schedule data produced by PF-NETS. 


\section{(6) PC Component Design System (FALCON)}

This system reads building product data in a standard format and supplements such data with information on electrical services, air conditioning, and plumbing systems. This information is used as an input to the design of the precast concrete components.

In the remainder of this paper, we will explain in detail two products that were developed to generate a computer model for buildings and to utilize this information for the selection and evaluation of construction and erection methods and equipment.

\section{Building Product Model Input System (FINE-MODELER)}

Buildings, from those with a few stories to high rises, and those with limited spaces to those with large spaces, and from factories to hotels, can be modeled as architectural abstractions. To use CIC, it is necessary to develop computer models for all of these various types of buildings. However, this task is relatively tedious and time consuming to accomplish using current systems. The problem is further aggravated because most programs require a level of detailed information that may not be available at the early stages of model development

For this reason, this system was developed with the goal of simplifying the data input requirements. It is targeted at mid-to-high rise buildings of about 15 stories. These buildings are constructed with steel-framed reinforced concrete (SRC), with construction methods such as HPC.

The system supports many variations in the shape and usage of precast concrete members, and was designed to handle buildings with linear and L-shaped floor plans. Roof shapes supported are for flat and gable roofs with conventional, partial precast, and full precast slabs.

The system operates through a dialogue with the user. The user specifies general project information such as number of bays, number of floors, etc., and then selects sectional information about members from a stored list. The system then generates the building's main members such as columns, girders, walls, floors, balconies, and external corridors. These members are represented as three-dimensional objects based on key plan information and sectional information. The system also generates the roof and foundation members and allows the user to modify information about the attributes of members such as type of concrete, slab construction method, dimensions of the opening, and degree of segmentation. Data belonging to the model developed by the FINE-MODELER system may also be used by the structural design system.

\section{Preliminary Construction Planning System (FINE-PLANNER)}

In construction projects, a few design modifications may greatly facilitate construction. Constructability reviews are therefore an important ingredient towards the goal of reducing project construction cost and duration. The FINE-PLANNER system strives to improve building designs by integrating constructability concepts.

FINE-PLANNER was developed to analyze fundamental problems associated with the construction of a building after the preliminary design is established. With FINE-PLANNER the user can analyze construction plans in three phases, based on the three-dimensional building input data obtained from FINE-MODELER. In Phase 1, the user identifies temporary roads and selects a crane, then specifies a construction plan. In Phase 2, the user divides the scope of work into zones and performs various calculations to determine the number of members required for each zone based on Phase 1 results. In 
Phase 3, the user evaluates a cycle schedule, then makes a master schedule and calculates project costs. At this point the user can return to Phase 2 or 3 to examine alternative plans under different conditions.

The FINE-PLANNER system can be used for a 15-story single building consisting of up to 20 units; or two buildings of equal size, housing 10 units or less on each story. Three crane classes of 80-ton, 100-ton, and 150-ton are available to the user. During crane selection, precast members that are used for shear walls (with girders), floor slabs, balconies, and outer corridors may be split if overweight.

To operate the system, the user identifies the crane's travel path and selects a crane class; next he/she evaluates the adequacy of the net lifting load of the selected crane. There are two ways to do this; the computer may automatically verify whether there are any parts that the selected crane cannot lift; or alternatively, the user may designate a member and verify whether the selected crane can lift it. If the crane is not capable of lifting a member, it is identified the user where a heavier crane can be selected or the member may be split. The system then develops a cross sectional drawing of the position of the crane relative to the building. This is to verify the adequacy of the net lifting load of the selected crane relative to the height of the building.

Next, the user divides the entire building into two construction zones, and the computer calculates (1) quantity of each precast member, (2) amount of cast-in-place concrete, (3) quantity of each form, and (4) amount of concrete needed for members according to construction zone, story, and member type, based on types, weights, and materials of precast members and their positions. Finally, these calculations are output in the Microsoft Excel spreadsheet format. By studying this output, the user can assess the adequacy of the work balance between the two construction zones.

\section{Development of the schedule}

The work schedule for one story in one construction zone is called "cycle schedule", because the building is constructed by repeating this procedure for each story in a construction zone. A cycle schedule is developed based on the number of days to complete a typical story in one construction zone, with the assumption that the same procedure will be repeated for each story of the construction zone.

The user first selects (1) the number of days required to complete one story, and (2) the nonbearing wall installation sequence, and adds to these the previously input (3) slab placement method. Then the user selects the applicable cycle scheduling pattern from the FINE-PLANNER system.

Cycle scheduling patterns are presented in the Excel format. The system also calculates the number of precast members to be erected, the amount of labor required for structural work, and the hours of crane utilization on a daily basis. The computer displays the cycle schedule along with these calculations. This enables the user to judge whether these selections are appropriate.

The next step is making a master schedule based on the established cycle schedule as shown in figure 2. The master schedule covers pre-erection (earth work, and foundation work), erection, and post-erection (equipment, finishing, and external work) processes. The process data generated is automatically transferred to the Construction Management System (PF-NETS). Here, the user can develop a more detailed schedule or utilize the data for progress assessment after the start of construction. 


\section{Estimating Erection Costs and Comparison of Alternative Plans}

Cost, an important factor when comparing and evaluating alternative plans under various work conditions, can also be calculated. Major factors affecting cost in alternative plans are (1) crane type duration use, (2) cycle scheduling (the amount of labor required for erection work). The system calculates the erection costs in detail, and this generated cost data can be used in Fujita's Estimating System after it is converted.

By performing the above steps, more than one alternative plan can be evaluated with FINE-PLANNER which also employs a Hyper Text structure where a table showing typical items for comparison appears on the monitor. By clicking on any item, more detailed information appears on the monitor.

\section{LIMITATIONS OF CURRENT APPROACHES}

To fully serve the multitude of information requirements needed by the various specializations in a building, it is an absolute necessity that an integrated project database be developed. The literature lists several attempts at the development of such a system.

Research in this area can be grouped in three general thrusts. The first thrust is involved with the development of a "standalone" framework that supports the full range of activities in a project, spanning from conceptual design and extending to operations and maintenance. While this approach is revolutionary, the majority of such systems cannot communicate easily with existing legacy systems thereby limiting their practical application. Another drawback is that once an object is specified, it becomes relatively cumbersome to re-engineer. The second thrust involves attempts to develop standardized methods for implementing objects in a project. These objects would then be capable of being exported and manipulated by existing legacy systems. This area may have the highest potential of application in the industry. However, these efforts are hampered by the lack of software vendor support although this is starting to change. The third thrust involves the development of graphical modeling representation languages for buildings.

The representation of modern buildings in the computer is a complex one. The problem is further complicated by the desire of researchers to create a single representation whose ultimate benefits, specifications, and requirements are not quite defined or known. This has caused the research to be following a "moving" target.

There are also other problems related to implementation in the computer. Some researchers prefer to base the system on a relational structure whereas others prefer to base it on an object oriented platform. Researchers have also been debating for a while the merits of both approaches.

The majority of objects in a construction project are related to other objects, which is referred to in the database literature as a many-to-many relationship. Figure 3 shows such a relationship between beam and column objects where each beam may be supported on multiple columns and each column may support multiple beams. The implementation of such a relationship in a relational database is also shown in figure 3 . This requires the database system to assemble the object whenever it is needed. These table "join" operations are time consuming. Also, the actual object and its relationships are stored across multiple tables. 
In engineering applications, there is typically a large number of tables with each table having only a few entries. This is exactly the opposite to objects in business applications which typically have a relatively few number of tables with each table having an extremely large number of entries. This means that in engineering applications, a very large number of joins is needed to assemble objects, in addition to storing these objects across multiple table boundaries. For these reasons, some researchers have advocated the use of object oriented databases where information related to objects is stored in contiguous locations thereby speeding data access. Access speed, however, is not the strongest reason for using an object oriented database. The real benefits are in the support for inheritance and object methods. Also another major benefit is the $1: 1$ mapping between the actual objects and their computer representations.

Object oriented databases remain niche players in the database markets. The benefits of their application has been outlined above, however their application in construction is not without its problems. In construction applications, objects tend to evolve and change as the project progresses. In some situations, an object may change form as in stripping a concrete form to expose the new wall (new object).

These seems to be a consensus from the research that the general notion of object representation is a better way to go. However, the past few years have demonstrated that inadequately designed objects, while solving some old problems, introduce there own anomalies. Also, business and other rules need to be stored within objects thereby limiting the flexibility and data reuse. It is therefore imperative that an extensive study of potential object representations and their intended used be done before the selection of hardware and software for the system is done.

The market has recently responded with CASE tools to assist the modeler in developing better object representations for systems. Some of these tools go beyond the graphical representation to the actual code that generates the underlying database schemas.

Another positive development is the creation of alliances for the standardization of object representations. This is an extremely positive development since the benefits of code reuse by developing object oriented systems is currently far from true. It remains extremely elusive to truly benefit from an "object-oriented " software library due to the lack of standardization between the various companies offering software solutions.

It appears that an object-based approach with mappings to legacy systems is the best interim solution. This allows construction companies to test and "tweak" their object implementations, and use their existing systems before transitioning to a new environment. The authors are continuing their research in the important area of object representations.

\section{ACKNOWLEDGMENTS}

The authors wish to thank the Fujita Corporation of Japan for the continued support for this research. 

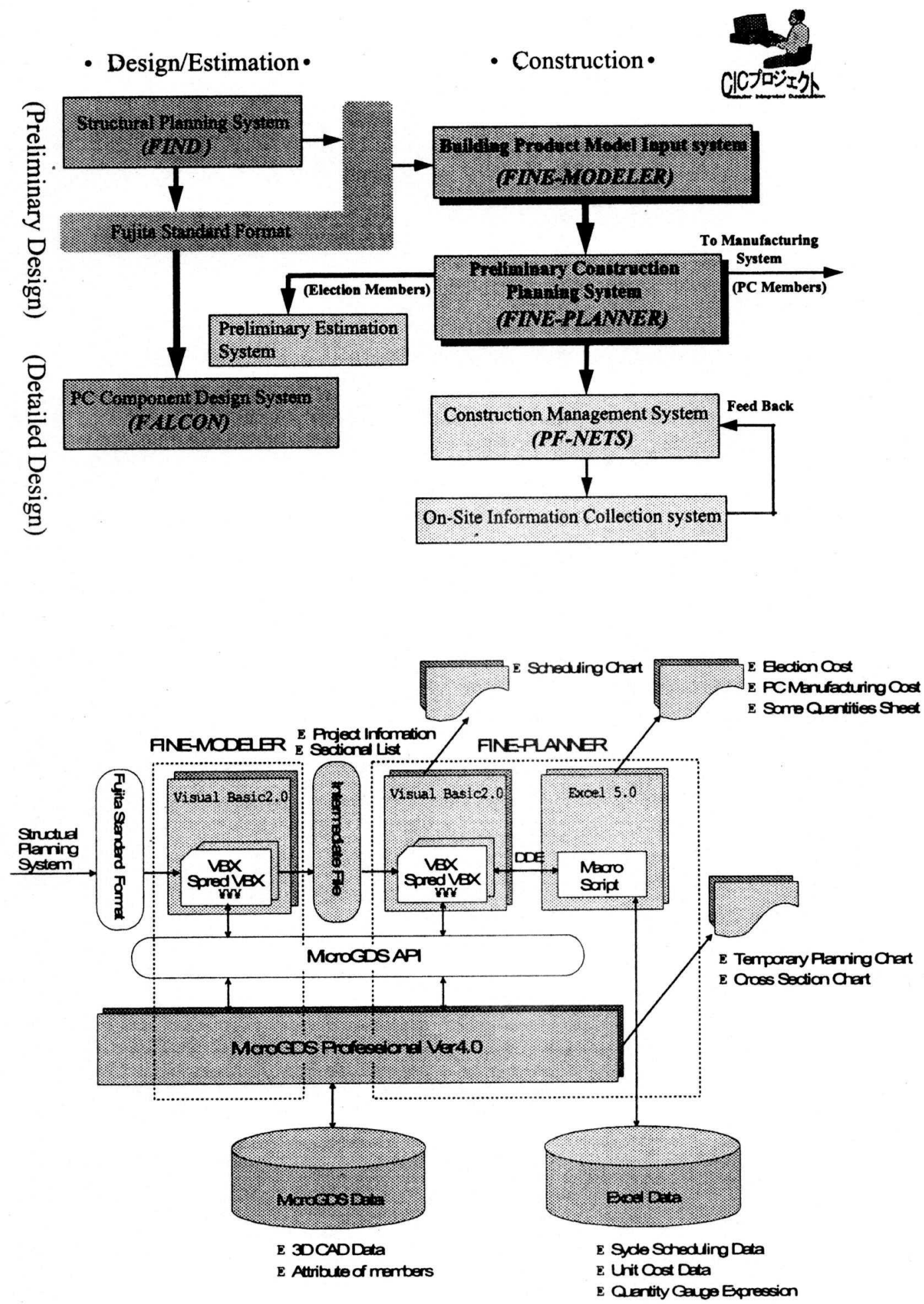

Figure 1 

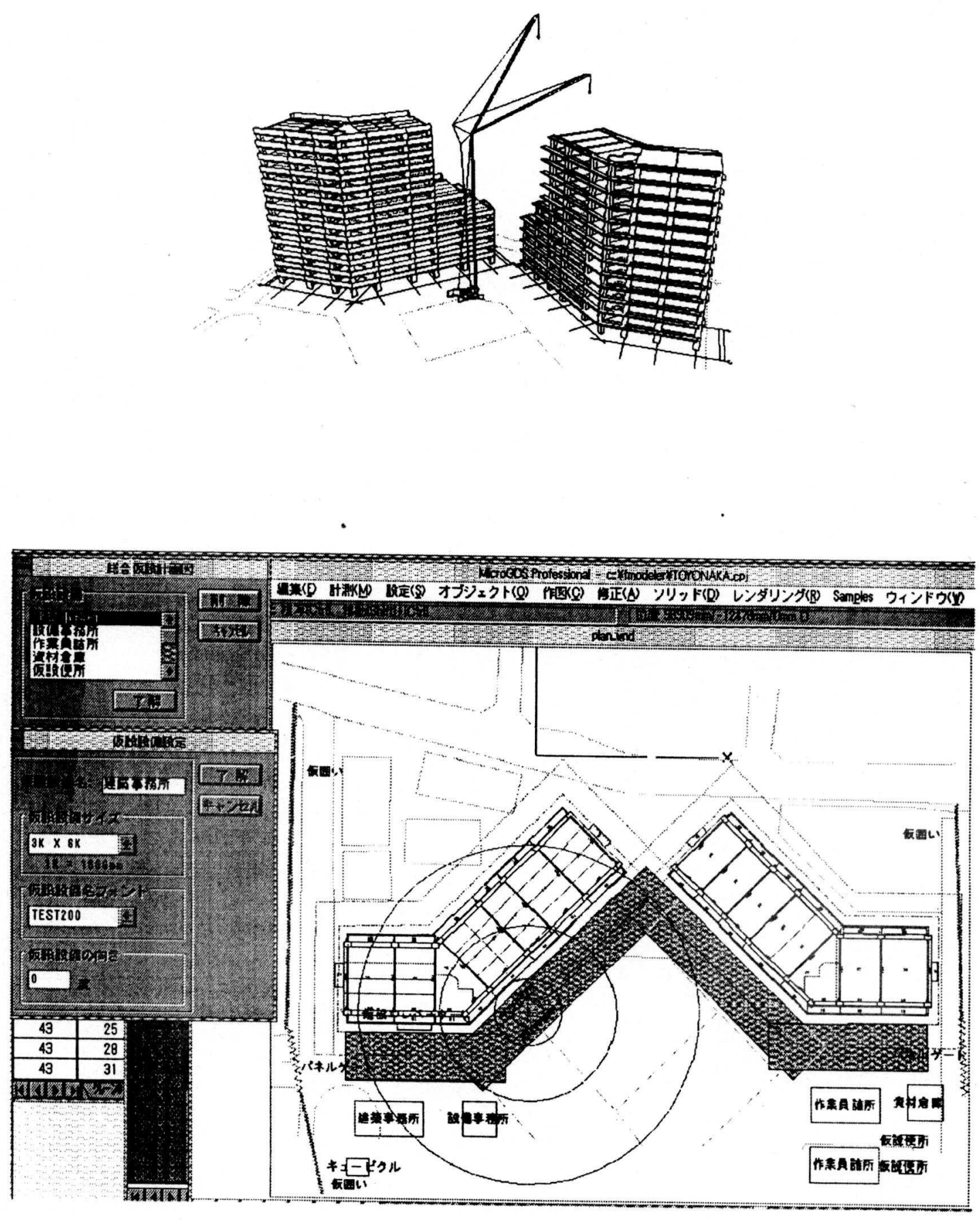

Figure 2 


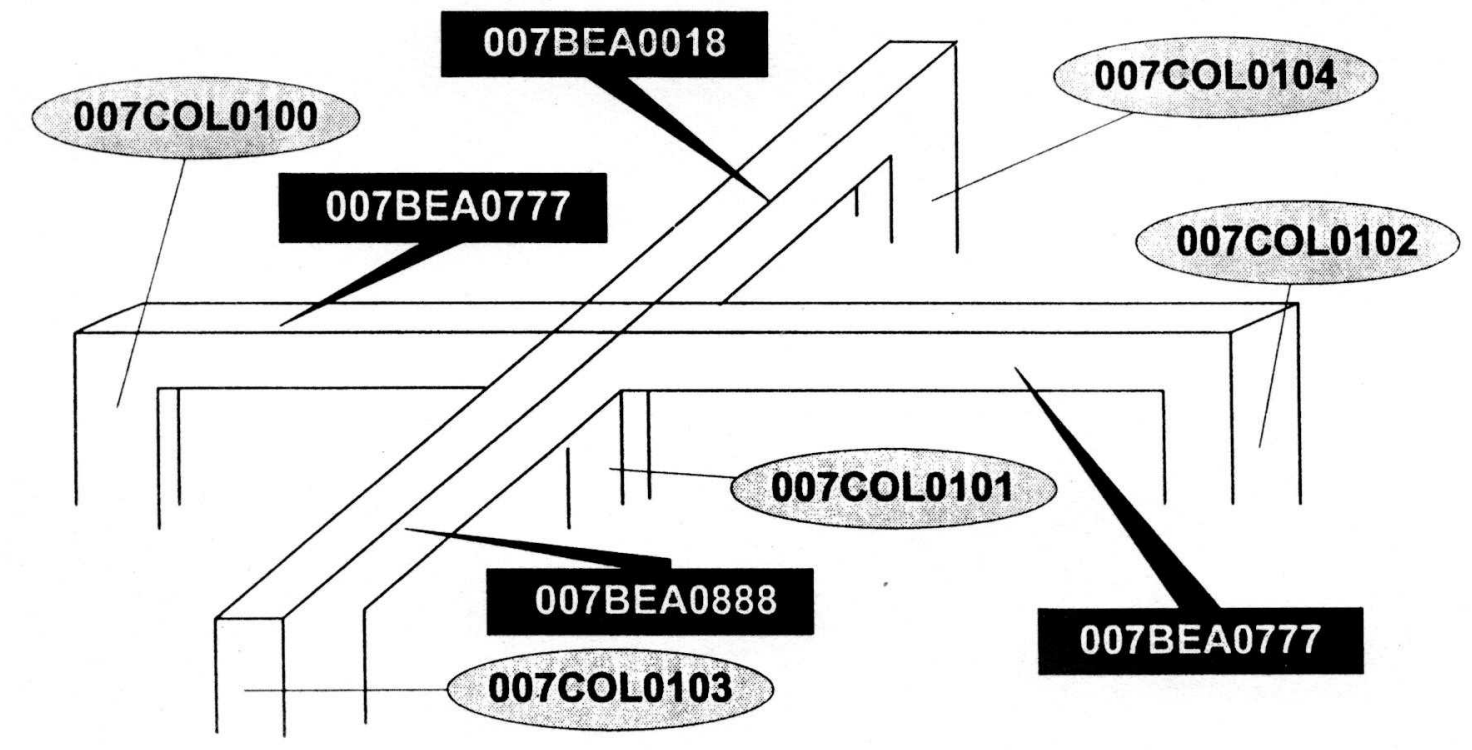

\section{BEAM}

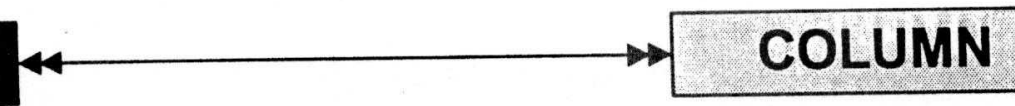

\section{BEAM}

\section{BF}

\section{COLUMN}

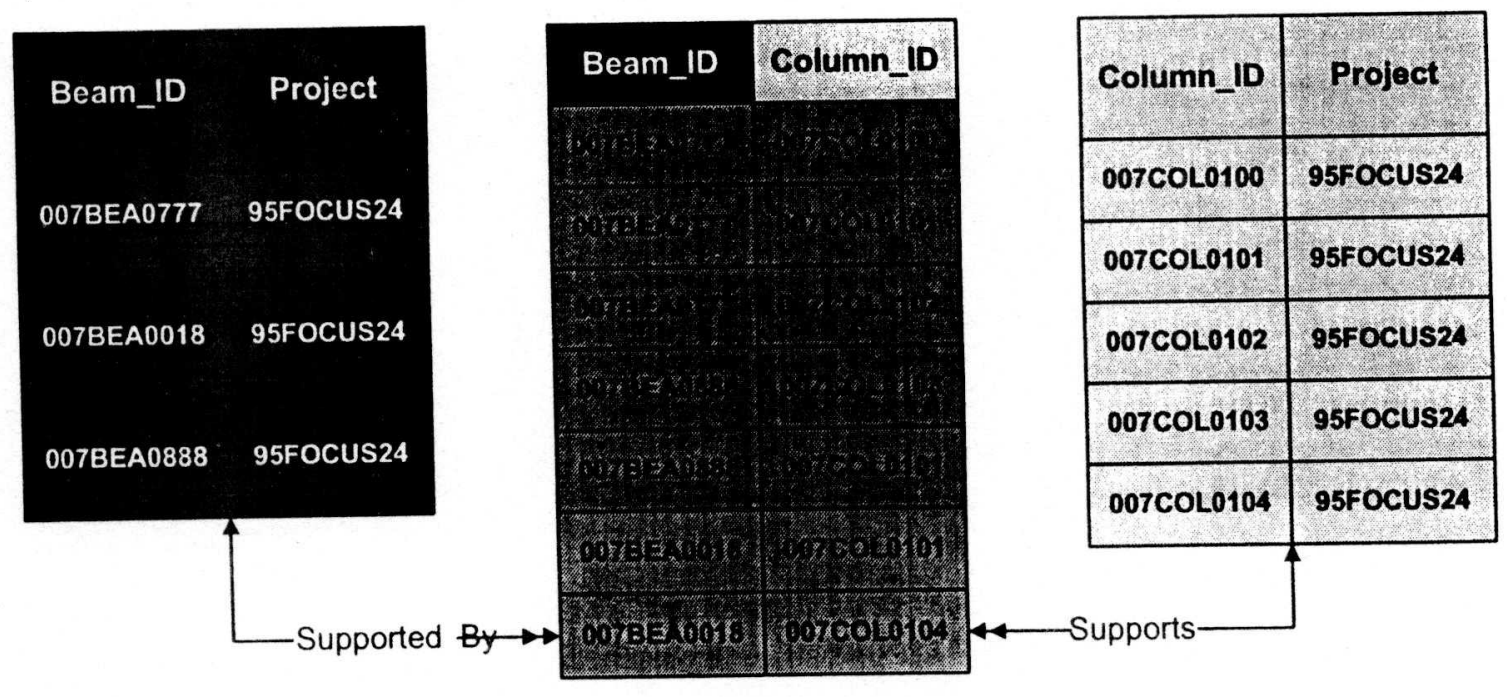

Figure 3

13th ISARC 